International Journal of Applied Mathematics

Volume 34 No. $6 \quad 2021,1153-1170$

ISSN: 1311-1728 (printed version); ISSN: 1314-8060 (on-line version)

doi: http://dx.doi.org/10.12732/ijam.v34i6.8

\title{
RELIABILITY STUDIES OF GENERALIZED SIBUYA DISTRIBUTION AND ITS APPLICATION
}

\author{
F. Alqallaf ${ }^{1} \S$, M.E. Ghitany ${ }^{1}$, \\ Ramesh C. Gupta ${ }^{2}$, J. Mazucheli ${ }^{3}$ \\ ${ }^{1}$ Department of Statistics and Operations Research \\ Faculty of Science, Kuwait University, KUWAIT \\ ${ }^{2}$ Department of Mathematics and Statistics \\ University of Maine, Orono, Maine, USA \\ ${ }^{3}$ Department of Statistics \\ Universidade Estadual de Maringá \\ Maringá, PR, BRAZIL
}

\begin{abstract}
In this paper, we have studied the generalized Sibuya distribution from a reliability point of view. It turns out that this distribution has the logconvex property and hence is infinitely divisible. This enables us to study the monotonic properties of various reliability functions including the failure rate, the mean residual life, the variance residual life and their reversed versions. The monotone properties of the classes of discrete distributions are parallel to those of continuous distributions. Procedures are developed to estimate the reliability functions. Application to real data is provided to illustrate the results.
\end{abstract}

AMS Subject Classification: $62 \mathrm{~N} 02,62 \mathrm{~N} 03,62 \mathrm{~N} 99,62 \mathrm{~F} 99$

Key Words: log-convexity; hazard rate; mean residual life; variance residual life; maximum likelihood

\section{Introduction}

Recently, in [9] a generalized Sibuya distribution $\left(\mathrm{GS}_{1} \mathrm{D}\right)$ is presented and some

Received: May 24, 2021

(c) 2021 Academic Publications

${ }^{\S}$ Correspondence author 
of its properties are studied. The distribution arises as the waiting time (discrete) for the first success in independent Bernoulli trials where the probability of success at each trial is inversely proportional to the quantity $\nu+j$ where $\nu \geq 0$ and $j$ is the trial number. More specifically, in a sequence of Bernoulli trials $\left\{I_{j}, j \in \mathbb{N}=\{1,2, \ldots\}\right\}$, the probability of success at trial $j$ is given by

$$
\mathbb{P}\left(I_{j}=1\right)=\frac{\alpha}{\nu+j}, \quad j \in \mathbb{N}=\{1,2, \ldots\}, \quad \nu \geq 0, \quad 0<\alpha<\nu+1
$$

The above authors also studied the distribution of $X-m$ given $X>m$, where $X$ has $\mathrm{GS}_{1} \mathrm{D}$ and $m \in \mathbb{N}_{0}=\{0,1,2, \ldots\}$. In reliability and survival analysis, $X-m$ is called the residual time or survival time where $X$ is measured in discrete times, see [5].

The $\mathrm{GS}_{1} \mathrm{D}$ has one important property of being log-convex, shown in Section 2. This log-convex property enables us to study the monotonicity of various functions in reliability and life testing. The log-concavity and log-convexity of functions and sequences in probability have been of great interest to several authors, see [7]. The log-concavity and log-convexity properties have played important roles in economics, social sciences, information theory, optimization and theory of reliability. The log-concavity of the continuous distributions has been extensively studied in the literature, see [2].

The purpose of this paper is to study the $\mathrm{GS}_{1} \mathrm{D}$ from a reliability point of view. The log-concave (log-convex) distributions have increasing failure rate (decreasing failure rate), abbreviated as IFR (DFR), and hence decreasing mean residual life (DMRL) and increasing mean residual life (IMRL). We study these, and some other reliability functions and their monotonicity. We also derive procedures for estimating these functions. For that, maximum likelihood estimation of the parameters is carried out along with some simulation studies to examine the performance of the estimators.

The contents of the paper are arranged as follows. In Section 2, we present the structural properties along with log-convexity property of the $\mathrm{GS}_{1} \mathrm{D}$. In Section 3, we study various reliability functions and their monotonicity. Section 4 contains the estimation of the various reliability functions. The simulation studies are carried out in Section 5 to examine the performance of the estimators. Section 6 contains data analysis and some examples. Finally, in Section 7 , we present some comments and concluding remarks. 


\section{The model and its properties}

A discrete random variable $X$ with support on $\mathbb{N}$ is said to have a generalized Sibuya distribution with parameters $\alpha$ and $\nu$, denoted by $G S_{1}(\alpha, \nu)$, if its probability mass function (PMF) is given by

$$
f(x)=\frac{\alpha}{\nu+x} \prod_{i=1}^{x-1}\left(1-\frac{\alpha}{\nu+i}\right)=\frac{\alpha}{\nu+x} \frac{(\nu+1-\alpha)_{x-1}}{(\nu+1)_{x-1}},
$$

where $x \in \mathbb{N}, \nu \geq 0,0<\alpha<\nu+1$, and

$$
(a)_{0}=1, \quad(a)_{k}=a(a+1)(a+2) \ldots(a+k-1), \quad k \in \mathbb{N},
$$

is the (rising) Pochhammer symbol. Note that $(a)_{k}=\frac{\Gamma(a+k)}{\Gamma(a)}$, for $a>0$, where $\Gamma(\cdot)$ is the gamma function. This discrete distribution was recently studied by [9]. Note that $\nu=0$ gives the Sibuya distribution [13] with PMF

$$
f(x)=\alpha \frac{(1-\alpha)_{x-1}}{x !}, \quad n \in \mathbb{N}, \quad 0<\alpha<1 .
$$

The PMF $f(x)$ satisfies the recurrence relation:

$$
f(x+1)=\frac{\nu+x-\alpha}{\nu+x+1} f(x), \quad x \in \mathbb{N},
$$

with $f(1)=\frac{\alpha}{\nu+1}$.

Since

$$
\frac{f(x+1)}{f(x)}=\frac{\nu+x-\alpha}{\nu+x+1}<1,
$$

it follows that $f(x)$ is strictly decreasing and hence the mode occurs at $x=1$.

The mean and variance of the $\mathrm{GS}_{1} \mathrm{D}$, respectively, are given by

$$
\begin{aligned}
\mu & =\frac{\nu}{\alpha-1}, \quad \nu>0, \quad 1<\alpha<\nu+1, \\
\sigma^{2} & =\frac{\alpha \nu(\nu+1-\alpha)}{(\alpha-1)^{2}(\alpha-2)}, \quad \nu>1, \quad 2<\alpha<\nu+1 .
\end{aligned}
$$

The index of dispersion of the $\mathrm{GS}_{1} \mathrm{D}$ with PMF (1) is given by

$$
\gamma=\frac{\sigma^{2}}{\mu}=\frac{\alpha(\nu+1-\alpha)}{(\alpha-1)(\alpha-2)}, \quad \nu>1, \quad 2<\alpha<\nu+1 .
$$

A distribution is said to be over-dispersed (under-dispersed) if $\gamma>1(\gamma<1)$.

We now show some important properties of $G S_{1} \mathrm{D}$. 
Theorem 1. GS 1 D is over-dispersed (under-dispersed) if $2<\alpha<\alpha_{1}\left(\alpha_{1}<\alpha<\nu+1\right)$, where

$$
2<\alpha_{1}=\frac{\nu+4+\sqrt{\nu(\nu+8)}}{4}<\nu+1 \text {. }
$$

Proof. For $2<\alpha<\nu+1$, we have

$$
\frac{\partial \gamma}{\partial \alpha}=-\frac{\nu\left(\alpha^{2}-2\right)-2(\alpha-1)^{2}}{(\alpha-1)^{2}(\alpha-2)^{2}}<0,
$$

since $\nu>\alpha-1>1$ and $\alpha^{2}-2>2(\alpha-1)>2$.

Therefore, $\gamma$ is a decreasing function in $\alpha$, with $\gamma \rightarrow \infty$ as $\alpha \rightarrow 2^{+}$and $\gamma \rightarrow 0$ as $\alpha \rightarrow(\nu+1)^{-}$. Moreover, for given $\nu>1$, the equation $\gamma=1$ implies

$$
2 \alpha_{1}^{2}-(\nu+4) \alpha_{1}+2=0, \quad \nu>1, \quad 2<\alpha_{1}<\nu+1,
$$

which has a unique solution

$$
2<\alpha_{1}=\frac{\nu+4+\sqrt{\nu(\nu+8)}}{4}<\nu+1 .
$$

Finally, $\gamma>1(\gamma<1)$ if $2<\alpha<\alpha_{1}\left(\alpha_{1}<\alpha<\nu+1\right)$.

Figure 1 shows the index of dispersion of $\mathrm{GS}_{1} \mathrm{D}$ as a function of $\alpha$ for selected values of $\nu$.

For a discrete distribution with $\operatorname{PMF} p(x)=\mathbb{P}(X=x)$, where $x \in \mathbb{N}_{0}$ or a subset of $\mathbb{N}_{0}, p(x)$ is log-concave (log-convex) if

$$
p^{2}(x+1)-p(x) p(x+2) \geq 0(\leq 0) .
$$

In [6] the log-concavity and log-convexity of $p(x)$ in terms of the sequence $\eta(x)=p(x+1) / p(x)$ have been studied.

Define

$$
\Delta \eta(x)=\eta(x)-\eta(x+1)=\frac{p(x+1)}{p(x)}-\frac{p(x+2)}{p(x+1)} .
$$

(i) If $\Delta \eta(x)>0$, the PMF $p(x)$ is log-concave and hence the distribution is IFR.

(ii) If $\Delta \eta(x)<0$, the PMF $p(x)$ is log-convex and hence the distribution is DFR.

(iii) If $\Delta \eta(x)=0$, the PMF $p(x)$ is given by $p(x)=p(0) c^{x}$, where $c$ is a constant. Here, there are three possibilities: geometric distribution (constant failure rate), discrete uniform distribution (IFR) and discrete power function (IFR), see [10], p. 172.

In the following we show that the $\mathrm{GS}_{1} \mathrm{D}$ is log-convex. 

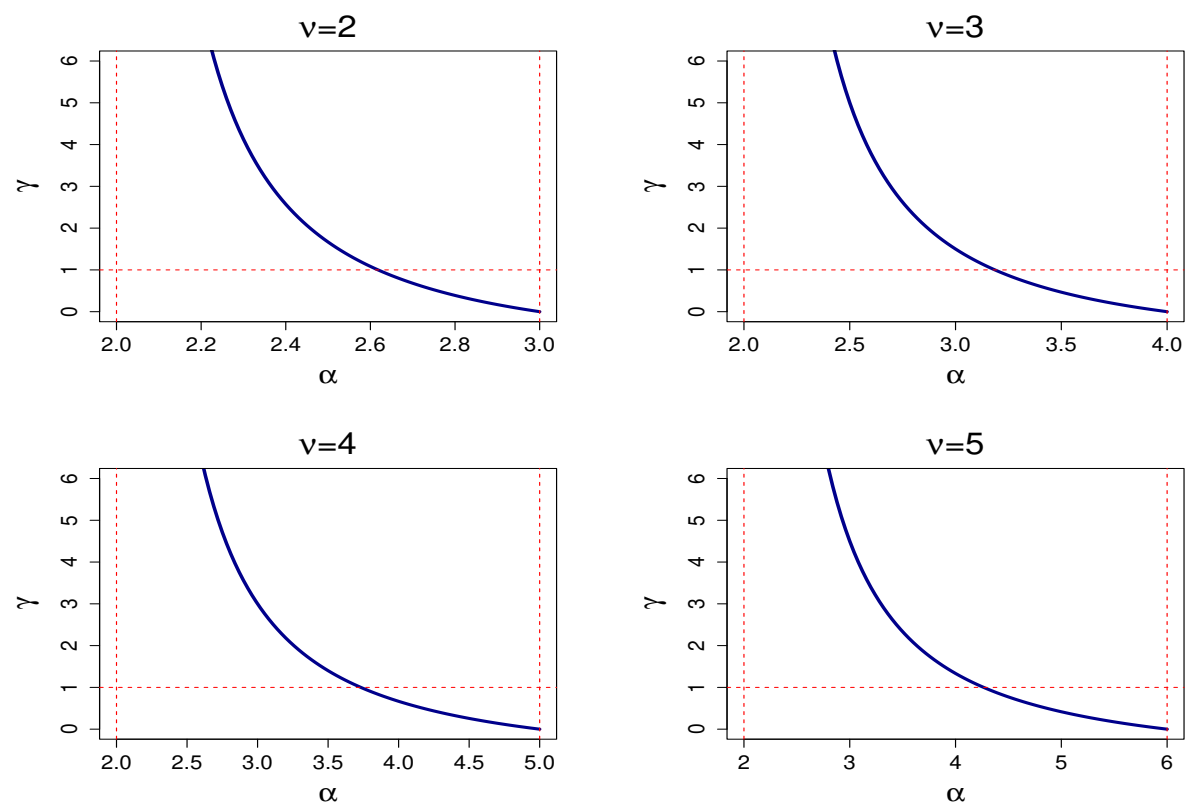

Figure 1: Index of dispersion of $\mathrm{GS}_{1}$ distribution as a function of $\alpha$ for selected values of $\nu$.

Theorem 2. $f(x)$ is log-convex.

Proof. For $x \in \mathbb{N}$,

$$
\Delta \eta(x)=\frac{\nu+x-\alpha}{\nu+x+1}-\frac{\nu+x+1-\alpha}{\nu+x+2}=\frac{-(\alpha+1)}{(\nu+x+1)(\nu+x+2)}<0 .
$$

Therefore, $f(x)$ is log-convex.

Theorem 2 implies that the Sibuya distribution $(\nu=0)$ is log-convex.

The log-convexity of $f(x)$ of $\mathrm{GS}_{1} \mathrm{D}$ implies that the distribution is DFR and hence IMRL. Also, GS ${ }_{1} \mathrm{D}$ is infinitely divisible, see [7]. The infinite divisibility property of $\mathrm{GS}_{1} \mathrm{D}$ was shown by [9], via its representation as mixture of geometric distribution, see p. 868.

\section{Reliability functions}

\subsection{Failure rate function}


Since the reliability function (survival function) is given by

$$
\bar{F}(x)=\mathbb{P}(X>x)=\prod_{i=1}^{x}\left(1-\frac{\alpha}{\nu+i}\right)=\frac{(\nu+1-\alpha)_{x}}{(\nu+1)_{x}},
$$

where $\nu \geq 0,0<\alpha<\nu+1$, it follows that the failure rate function (FRF) is given by

$$
h(x)=\mathbb{P}(X=x \mid X \geq x)=\frac{f(x)}{\bar{F}(x-1)}=\frac{\alpha}{\nu+x},
$$

where $x \in \mathbb{N}$ and $\nu \geq 0,0<\alpha<\nu+1$. Clearly, $h(x)$ is a decreasing function in $x$. This property also follows from the fact that $\mathrm{GS}_{1} \mathrm{D}$ is log-convex.

Note that the Sibuya distribution (i.e. $\nu=0$ ) has FRF:

$$
h(x)=\frac{\alpha}{x}, \quad x \in \mathbb{N}, \quad 0<\alpha<1 .
$$

For continuous time distribution, the FRF (also known as hazard rate function) is considered in [1] and [4].

\subsection{Reversed failure rate function}

The reversed failure rate function (RFRF) of the $G S_{1}(\alpha, \nu)$ is given by

$$
h^{*}(x)=\mathbb{P}(X=x \mid X \leq x)=\frac{f(x)}{F(x)}=\frac{\alpha(\nu+1-\alpha)_{x-1}}{(\nu+1)_{x}-(\nu+1-\alpha)_{x}},
$$

where $x \in \mathbb{N}$ and $\nu \geq 0,0<\alpha<\nu+1$. Note that $h^{*}(1)=1$, since $f(1)=F(1)$.

Since $\mathrm{GS}_{1} \mathrm{D}$ is log-convex, $h^{*}(x)$ is decreasing in $x$.

Note that the Sibuya distribution (i.e. $\nu=0$ ) has RFRF:

$$
h^{*}(x)=\frac{\alpha(1-\alpha)_{x-1}}{x !-(1-\alpha)_{x}}, \quad x \in \mathbb{N}, \quad 0<\alpha<1 .
$$

\subsection{Mean residual life function}

In [9] it is shown that the conditional distribution of $X-m$ given $X>m$, where $m \in \mathbb{N}_{0}$, is again $\mathrm{GS}_{1} \mathrm{D}$ with parameters $\alpha$ and $\nu+m$. Specifically, for $m \in \mathbb{N}_{0}$,

$$
X-m \mid X>m \sim G S_{1}(\alpha, \nu+m), \quad \nu \geq 0, \quad 0<\alpha<\nu+m+1,
$$

see Proposition 3, p. 860, of [9].

Using equation (5), the mean residual life function (MRLF) of the $\mathrm{GS}_{1} \mathrm{D}$ is given by

$$
\mu(x)=\mathbb{E}(X-x \mid X \geq x)=\frac{\nu+x-1}{\alpha-1}-1
$$


where $x \in \mathbb{N}$ and $\nu>0,1<\alpha<\nu+x$.

Clearly, $\mu(x)$ is increasing in $x$. This property also follows from the fact that DFRF $h(x)$ implies IMRLF $\mu(x)$. Also, $\mu(x)$ does not exist for Sibuya distribution $(\nu=0)$, since its mean does not exist.

For continuous time distribution, the MRLF is considered in [1].

\subsection{Reversed mean residual life function}

The reversed mean residual life function (RMRLF) of $G S_{1}(\alpha, \nu)$ is given by

$$
\begin{aligned}
\mu^{*}(x) & =\mathbb{E}(x-X \mid X \leq x) \\
& =\frac{\sum_{i=1}^{x} F(i-1)}{F(x)} \\
& =\frac{x-\frac{\nu}{\alpha-1}\left[1-\frac{(\nu+1-\alpha)_{x}}{(\nu)_{x}}\right]}{1-\frac{(\nu+1-\alpha)_{x}}{(\nu+1)_{x}}},
\end{aligned}
$$

where $x \in \mathbb{N}$ and $\nu>0,1<\alpha<\nu+1$. Note that $\mu^{*}(1)=0$.

Since $h^{*}(x)$ is decreasing, then $\mu^{*}(x)$ is increasing, see [5], Theorem 3.1, p. 4120.

Note that $\mu^{*}(x)$ does not exist for Sibuya distribution $(\nu=0)$, since its mean does not exist.

\subsection{Variance residual life}

Using equation (5), the variance residual life function (VRLF) of the $G S_{1}(\alpha, \nu)$ distribution is given by

$$
\sigma^{2}(x)=\operatorname{Var}(X-x \mid X \geq x)=\frac{\alpha(\nu+x-1)(\nu+x-\alpha)}{(\alpha-1)^{2}(\alpha-2)},
$$

where $x \in \mathbb{N}$ and $\nu>1,2<\alpha<\nu+x$.

Clearly, $\sigma^{2}(x)$ is increasing in $x$. This property also follows from the fact that IMRLF $\mu(x)$ implies IVRLF $\sigma^{2}(x)$, see [5], Theorem 2.2, p. 4118. Also, note that $\sigma^{2}(1)=\sigma^{2}$.

Note that for Sibuya distribution, VRLF does not exist since its variance does not exist.

\subsection{Reversed variance residual life}

The reversed variance residual life function (RVRLF) of the $G S_{1}(\alpha, \nu)$ distribution is given by

$$
\sigma^{* 2}(x)=\operatorname{Var}(x-X \mid X \leq x)
$$




$$
=(2 x+1) \mu^{*}(x)-\left[\mu^{*}(x)\right]^{2}-\frac{2 \sum_{i=1}^{x} i F(i-1)}{F(x)},
$$

where $x \in \mathbb{N}, \mu^{*}(x)$ is the RMRLF (7) (See [5] pp. 4119-4120) and

$$
\begin{aligned}
\sum_{i=1}^{x} i F(i-1)= & \frac{x(x+1)}{2} \\
& +\frac{(\nu+x)[x(\alpha-1)+\nu-1] \bar{F}(x)-\nu(\nu-1)}{(\alpha-1)(\alpha-2)} .
\end{aligned}
$$

Note that $\sigma^{* 2}(1)=\operatorname{Var}(1-X \mid X \leq 1)=0$.

Since IRMRLF implies IRVARF, see [5] Theorem 3.2, p. 4121, it follows that $\sigma^{* 2}(x)$ is increasing in $x \in \mathbb{N}$.

\section{Maximum likelihood estimation}

Let $x_{1}, x_{2}, \ldots, x_{n}$ be a random sample of size $n$ from $G S_{1}(\alpha, \nu)$ distribution. We assume that there exists at least one $x_{i}>1, i=1,2, \ldots, n$. The log-likelihood function is given by

$$
\begin{aligned}
\ell_{n}(\alpha, \nu)= & \sum_{i=1}^{n} \ln f\left(x_{i}\right) \\
= & n \ln (\alpha)-\sum_{i=1}^{n} \ln \left(\nu+x_{i}\right) \\
& +\sum_{i=1}^{n} \sum_{j=1}^{x_{i}-1}[\ln (\nu+j-\alpha)-\ln (\nu+j)] \\
= & n \ln (\alpha)-\sum_{i=1}^{n} \sum_{j=1}^{x_{i}} \ln (\nu+j) \\
& +\sum_{i=1}^{n} \sum_{j=1}^{x_{i}-1} \ln (\nu+j-\alpha) .
\end{aligned}
$$

It follows that the maximum-likelihood estimators (MLEs) $(\widehat{\alpha}, \widehat{\nu})$ of the parameters $(\alpha, \nu)$ are the simultaneous solutions of the equations:

$$
\frac{\partial \ell_{n}}{\partial \alpha}=\frac{n}{\alpha}-\sum_{i=1}^{n} \sum_{j=1}^{x_{i}-1} \frac{1}{\nu+j-\alpha}=0
$$




$$
\frac{\partial \ell_{n}}{\partial \nu}=-\sum_{i=1}^{n} \sum_{j=1}^{x_{i}} \frac{1}{\nu+x_{i}}+\sum_{i=1}^{n} \sum_{j=1}^{x_{i}-1} \frac{1}{\nu+j-\alpha}=0
$$

The solution of (11) and (12) leads to

$$
\widehat{\alpha}=\frac{n}{\sum_{i=1}^{n} \sum_{j=1}^{x_{i}} \frac{1}{\widehat{\nu}+j}}=\eta_{1}(\widehat{\nu}), \quad \text { say },
$$

where $\widehat{\nu}$ is the solution of the non-linear equation:

$$
\frac{n}{\eta_{1}(\widehat{\nu})}-\sum_{i=1}^{n} \sum_{j=1}^{x_{i}-1} \frac{1}{\widehat{\nu}+j-\eta_{1}(\widehat{\nu})}=0
$$

The second derivatives of the log-likelihood function are given by

$$
\begin{aligned}
\frac{\partial^{2} \ell_{n}}{\partial \alpha^{2}} & =-\frac{n}{\alpha^{2}}-\sum_{i=1}^{n} \sum_{j=1}^{x_{i}-1} \frac{1}{(\nu+j-\alpha)^{2}} \\
\frac{\partial^{2} \ell_{n}}{\partial \nu^{2}} & =\sum_{i=1}^{n} \sum_{j=1}^{x_{i}} \frac{1}{(\nu+j)^{2}}-\sum_{i=1}^{n} \sum_{j=1}^{x_{i}-1}\left[\frac{1}{(\nu+j-\alpha)^{2}}\right] \\
\frac{\partial^{2} \ell_{n}}{\partial \alpha \partial \nu} & =\sum_{i=1}^{n} \sum_{j=1}^{x_{i}-1} \frac{1}{(\nu+j-\alpha)^{2}} .
\end{aligned}
$$

The expected information matrix based on a single observation is given by

$$
\mathbf{I}(\alpha, \nu)=\left[I_{i j}(\alpha, \nu)\right], \quad i, j=1,2,
$$

where

$$
\begin{aligned}
& I_{11}(\alpha, \nu)=\frac{1}{\alpha^{2}}+C_{1}(\alpha, \nu), \\
& I_{22}(\alpha, \nu)=C_{1}(\alpha, \nu)-C_{2}(\alpha, \nu), \\
& I_{12}(\alpha, \nu)=-C_{1}(\alpha, \nu),
\end{aligned}
$$

with

$$
C_{1}(\alpha, \nu)=\mathbb{E}\left[\sum_{j=1}^{X-1} \frac{1}{(\nu+j-\alpha)^{2}}\right]
$$




$$
\begin{aligned}
= & \mathbb{E}\left[\psi^{\prime}(\nu+1-\alpha)-\psi^{\prime}(\nu+X-\alpha)\right] \\
= & \psi^{\prime}(\nu+1-\alpha) \\
& \quad-\sum_{x=1}^{\infty} \psi^{\prime}(\nu+x-\alpha) \frac{\alpha}{\nu+x} \frac{(\nu+1-\alpha)_{x-1}}{(\nu+1)_{x-1}},
\end{aligned}
$$

and

$$
\begin{aligned}
C_{2}(\alpha, \nu) & =\mathbb{E}\left[\sum_{j=1}^{X} \frac{1}{(\nu+j)^{2}}\right] \\
& =\mathbb{E}\left[\psi^{\prime}(\nu+1)-\psi^{\prime}(\nu+1+X)\right] \\
& =\psi^{\prime}(\nu+1)-\sum_{x=1}^{\infty} \psi^{\prime}(\nu+1+x) \frac{\alpha}{\nu+x} \frac{(\nu+1-\alpha)_{x-1}}{(\nu+1)_{x-1}}
\end{aligned}
$$

with $\psi^{\prime}(z)=\frac{d^{2}}{d z^{2}} \ln \Gamma(z)$ the digamma function.

Under mild regularity conditions (see [11], pp. 461-463), we have the following asymptotic result:

$$
\sqrt{n}\left(\left(\begin{array}{l}
\widehat{\alpha} \\
\widehat{\nu}
\end{array}\right)-\left(\begin{array}{l}
\alpha \\
\nu
\end{array}\right)\right) \stackrel{d}{\rightarrow} N_{2}\left(\mathbf{0}, \mathbf{I}^{-1}(\alpha, \nu)\right)
$$

where $N_{2}(\mathbf{0}, \boldsymbol{\Sigma})$ denotes the bivariate normal distribution with mean $\mathbf{0}$ and variance-covariance matrix $\boldsymbol{\Sigma}$.

Since the reliability functions, considered in Section 3, are real-valued functions in the parameters, say $g(x ; \alpha, \nu)$, we can use the delta-method to obtain

$$
\sqrt{n}(g(x ; \widehat{\alpha}, \widehat{\nu}))-g(x ; \alpha, \nu)) \stackrel{d}{\rightarrow} N\left(0, \tau^{2}\right) .
$$

where

$$
\tau^{2}=\mathbf{D}^{\top}(x, \alpha, \nu) \mathbf{I}^{-1}(\alpha, \nu) \mathbf{D}(x, \alpha, \nu)
$$

with

$$
\mathbf{D}^{\top}(x, \alpha, \nu)=\left(\frac{\partial g(x, \alpha, \nu)}{\partial \alpha}, \frac{\partial g(x, \alpha, \nu)}{\partial \nu}\right)
$$




\section{Monte Carlo simulation study}

In this section, we evaluate the performance of the maximum likelihood estimators and their asymptotic normality through a Monte Carlo simulation study.

To generate data from the $\mathrm{GS}_{1} \mathrm{D}$, we used the mixed Poisson representation:

$$
\begin{gathered}
X \mid U=\lambda \sim 1+\operatorname{Poisson}(\lambda), \\
U \stackrel{d}{=} \frac{T_{1} T_{2}}{T_{3}},
\end{gathered}
$$

where $T_{1} \sim \operatorname{Exponential}(1), T_{2} \sim \operatorname{Gamma}(\nu+1-\alpha, 1)$ and $T_{3} \sim \operatorname{Gamma}(\alpha, 1)$ are independent random variables. See Proposition 7 of [9], p. 865.

The simulation experiment was repeated $N=10,000$ times each with sample size

$$
n=50,100,150,200,250,300 .
$$

The true values of the parameters $(\alpha, \nu)$ are chosen as

$$
(0.5,0),(0.5,0.5),(1,0.5),(1.5,1),(1.5,2),(2,1.5),(2.5,2),(2.5,3) \text {. }
$$

Note that for cases 1-3 $(\alpha \leq 1), \mu$ does not exist, for cases $4-6(1<\alpha \leq 2), \mu$ only exists, and for cases 7-8 $(\alpha>2), \sigma^{2}$ exists.

All simulations were performed in $0 \mathrm{x}$ Console, version 8.02, [3], using the $M a x B F G S$ function to obtain the maximum likelihood estimates of the parameters $\alpha$ and $\nu$.

The evaluation of point estimation was performed based on the bias

$$
\operatorname{Bias}(\widehat{\theta})=\frac{1}{N} \sum_{i=1}^{N}(\widehat{\theta}-\theta), \quad \theta=\alpha, \nu,
$$

and mean squared error (MSE)

$$
M S E(\widehat{\theta})=\frac{1}{N} \sum_{i=1}^{N}(\widehat{\theta}-\theta)^{2}, \quad \theta=\alpha, \nu,
$$

for each sample size $n$. The estimated biases and MSEs of $(\widehat{\alpha}, \widehat{\nu})$ versus $n$ are shown in Figure 2. The biases are positive. The biases and MSEs decrease with increasing $n$.

The evaluation of interval estimation was performed based on the coverage probability $(\mathrm{CP})$ of $95 \%$ confidence intervals of each parameter and the average width (AW) 95\% confidence intervals of each parameter for each sample size. 

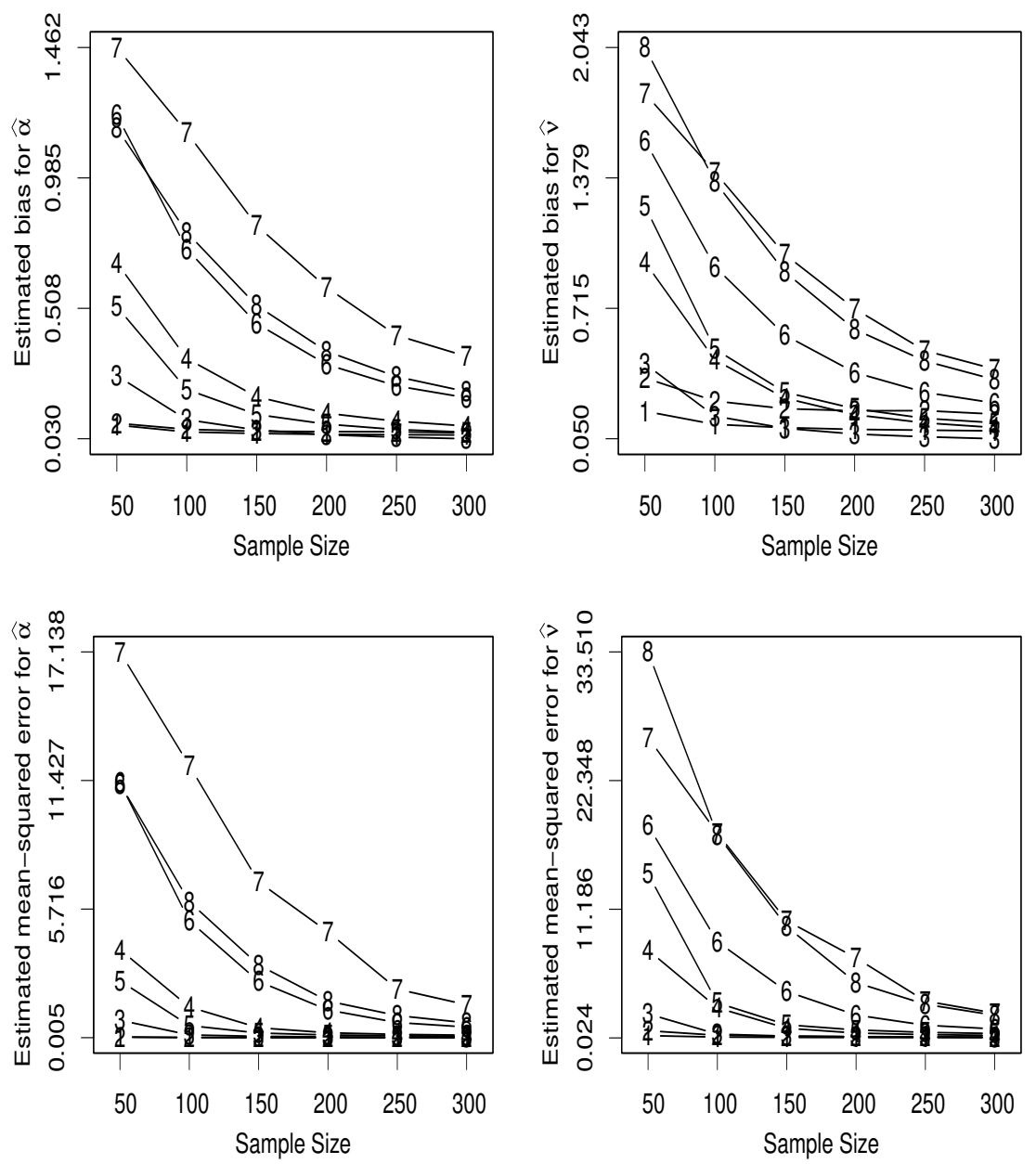

Figure 2: Estimated bias (upper panel) and estimated mean-squared error (lower panel) of the MLEs $\widehat{\alpha}$ and $\widehat{\nu}$. 1: $(\alpha=0.5, \nu=0.0), 2$ : $(\alpha=0.5, \nu=0.5), 3:(\alpha=1.0, \nu=0.5)$, 4: $(\alpha=1.5$, $\nu=1.0), 5:(\alpha=1.5, \nu=2.0), 6:(\alpha=2.0, \nu=1.5), 7:(\alpha=2.5$, $\nu=2.0)$ and $8:(\alpha=2.5, \nu=3.0)$.

The CPs and AWs of 95\% CIs of $\alpha$ and $\lambda$ versus $n$ are shown in Figure 3 . This figure shows that the coverage probabilities of the confidence intervals are close to the nominal level of $95 \%$ and that the average confidence widths decrease as the sample size increases, as one would expect. 

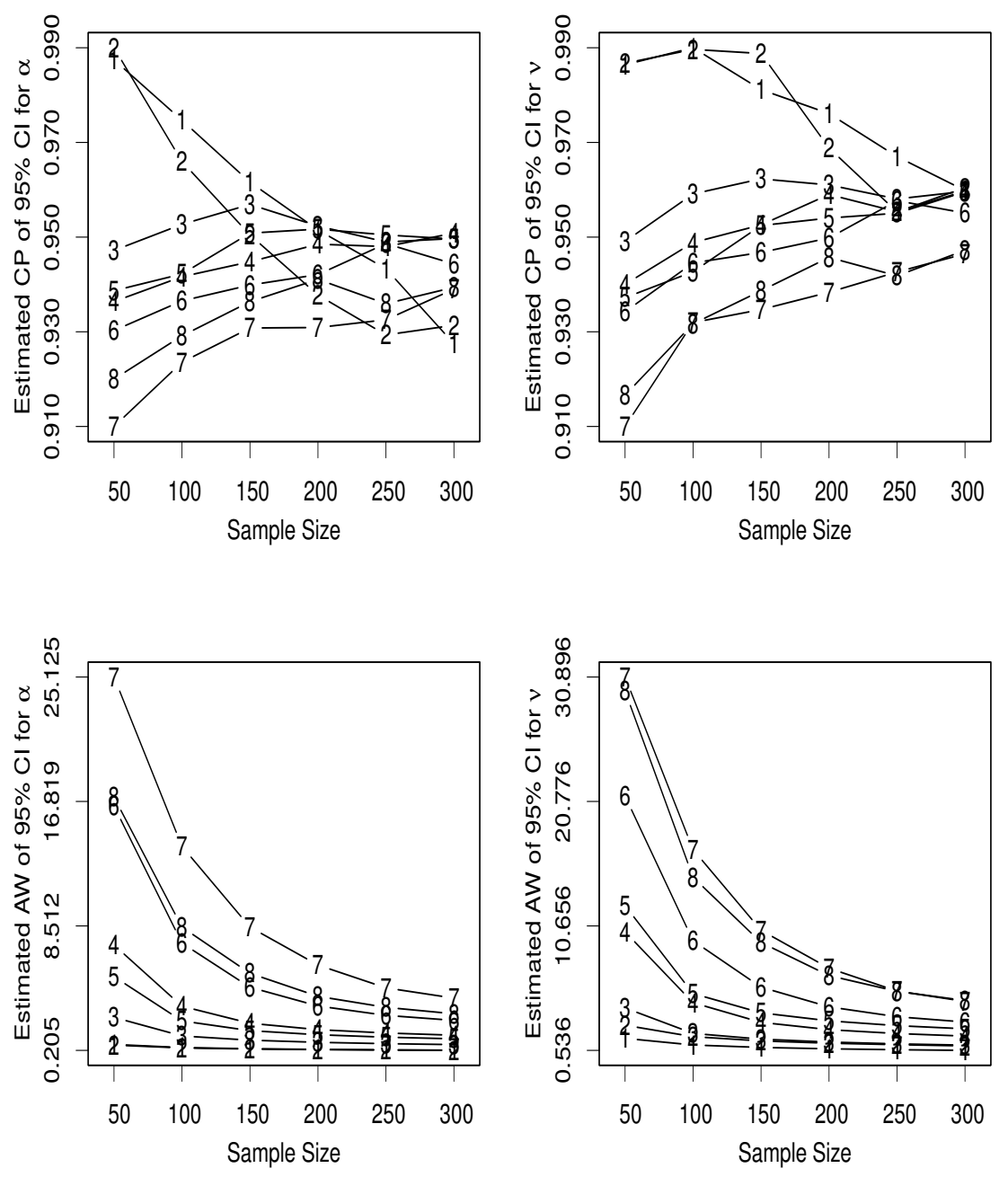

Figure 3: Estimated coverage probability (CP) of $95 \%$ confidence interval of $\alpha$ and $\nu$ (upper panel) and estimated average width (AW) of $95 \%$ confidence interval of $\alpha$ and $\nu$ (lower panel). $1:(\alpha=0.5$, $\nu=0.0), 2:(\alpha=0.5, \nu=0.5), 3:(\alpha=1.0, \nu=0.5), 4:(\alpha=1.5$, $\nu=1.0), 5:(\alpha=1.5, \nu=2.0), 6:(\alpha=2.0, \nu=1.5), 7:(\alpha=2.5$, $\nu=2.0)$ and $8:(\alpha=2.5, \nu=3.0)$.

\section{Data analysis}

In this section, we analyze a real data set represents 213 observations on the number of successive failures of the air conditioning system of a fleet of 13 
Boeing 720 jet airplanes, see [12].

Table 1 shows the maximum likelihood estimates (their standard errors) and the estimated log-likelihood function for Sibuya and generalized Sibuya distributions.

Table 1: MLEs (standard errors), estimated log-likelihood for Sibuya and and $G S_{1}$ distributions.

\begin{tabular}{|l|l|c|}
\hline Distribution & MLE (S.E.) & log-likelihood \\
\hline \hline Sibuya & $\widehat{\alpha}=0.207(0.014)$ & -1370.957 \\
\hline GS $_{1}$ & $\begin{array}{l}\widehat{\alpha}=5.753(2.908) \\
\end{array}$ & -1175.043 \\
& $\widehat{\nu}=444.911(261.562)$ & \\
\hline
\end{tabular}

Table 2: Observed frequency $(O)$ and expected frequency $(E)$ for Sibuya and $G S_{1}$ distributions.

\begin{tabular}{|l|c|c|c|}
\hline class & $O$ & $E$ (Sibuya) & $E\left(\mathrm{GS}_{1}\right)$ \\
\hline \hline $0-49$ & 97 & 131.75 & 96.59 \\
$50-99$ & 51 & 10.92 & 49.74 \\
$100-149$ & 21 & 5.68 & 26.56 \\
$150-199$ & 14 & 3.74 & 14.96 \\
$200-249$ & 14 & 2.75 & 8.82 \\
$\geq 250$ & 16 & 58.16 & 16.33 \\
\hline Total & 213 & 213 & 213 \\
\hline
\end{tabular}

Table 3 shows summary of Chi-square goodness-of-fit test for Sibuya and $G S_{1}$ distributions. Based on Table 3, we reject the null hypothesis that data is drawn from Sibuya distribution and fail to reject the null hypothesis that data is drawn from $G S_{1}$ distribution.

Table 3: Chi-square goodness-of-fit test for Sibuya and $G S_{1}$ distributions.

\begin{tabular}{|l|l|c|c|}
\hline Distribution & d.f. & $\chi^{2}$ statistic & $p$-value \\
\hline \hline Sibuya & 4 & 302.32 & 0.000 \\
\hline $\mathrm{GS}_{1}$ & 3 & 4.31 & 0.230 \\
\hline
\end{tabular}

In the following, we present the point estimation and $95 \%$ confidence interval of the reliability measures: MRLF $\mu(x)$ and $\operatorname{VRLF} \sigma^{2}(x)$ for given value $x=1,2, \ldots$ 

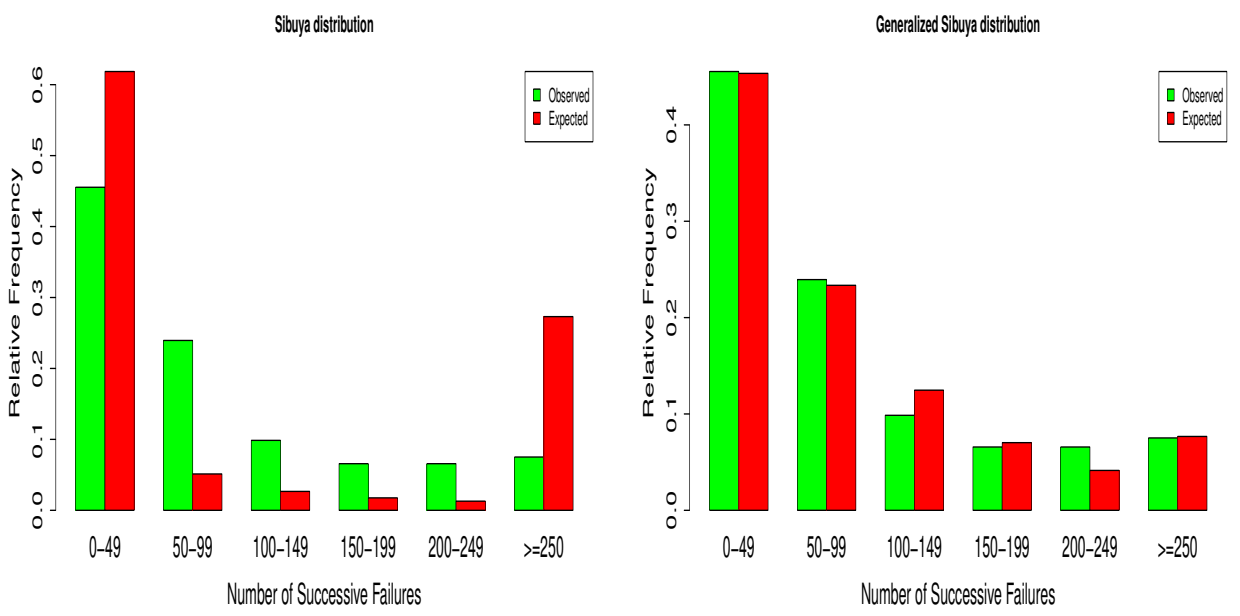

Figure 4: Observed versus expected relative frequency of Sibuya and Generalized Sibuya distributions for Proschan data.
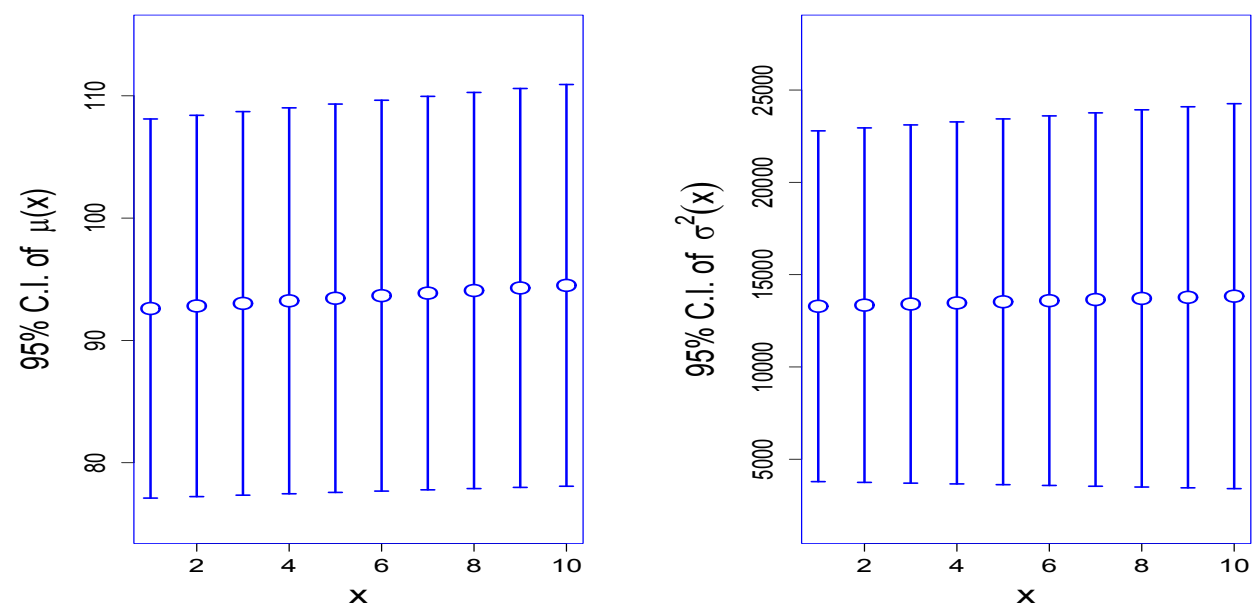

Figure 5: Confidence intervals of MRLF $\mu(x)$ and $\operatorname{VRLF} \sigma^{2}(x)$ for Proschan data.

Note that $\widehat{\mu}=\widehat{\mu(1)}+1=93.6$, S.E. $(\widehat{\mu})=$ S.E. $(\widehat{\mu(1)})=7.9$ and $95 \%$ C.I. of $\mu$ is $(78.1,109.1)$.

Note that $\widehat{\sigma^{2}}=\widehat{\sigma^{2}(1)}=13288.1$, S.E. $\left(\widehat{\sigma^{2}}\right)=$ S.E. $\left(\widehat{\sigma^{2}(1)}\right)=4847.4$ and $95 \%$ C.I. of $\sigma^{2}$ is $(3787.2,22788.9)$. 
Table 4: Point and interval estimation of MRLF $\mu(x)$.

\begin{tabular}{|c|c|c|c|c|}
\hline$x$ & $\widehat{\mu(x)}$ & S.E. $(\widetilde{\mu(x)})$ & $95 \%$ C.I. of $\mu(x)$ & C.I. width \\
\hline \hline 1 & 92.6 & 7.9 & $(77.1,108.1)$ & 31.0 \\
2 & 92.8 & 8.0 & $(77.2,108.4)$ & 31.2 \\
3 & 93.0 & 8.0 & $(77.3,108.7)$ & 31.4 \\
4 & 93.2 & 8.1 & $(77.5,109.0)$ & 31.6 \\
5 & 93.4 & 8.1 & $(77.6,109.3)$ & 31.8 \\
6 & 93.7 & 8.2 & $(77.7,109.6)$ & 32.0 \\
7 & 93.9 & 8.2 & $(77.8,110.0)$ & 32.2 \\
8 & 94.1 & 8.3 & $(77.9,110.3)$ & 32.4 \\
9 & 94.3 & 8.3 & $(78.0,110.6)$ & 32.6 \\
10 & 94.5 & 8.4 & $(78.1,110.9)$ & 32.8 \\
\hline
\end{tabular}

Table 5: Point and interval estimation of $\operatorname{VRLF} \sigma^{2}(x)$.

\begin{tabular}{|c|c|c|c|c|}
\hline$x$ & $\widehat{\sigma^{2}(x)}$ & S.E. $\left(\widehat{\sigma^{2}(x)}\right)$ & 95\% C.I. of $\sigma^{2}(x)$ & C.I. width \\
\hline \hline 1 & 13288.1 & 4847.4 & $(3787.2,22788.9)$ & 19001.7 \\
2 & 13348.2 & 4898.8 & $(3746.6,22949.8)$ & 19203.2 \\
3 & 13408.5 & 4950.4 & $(3705.6,23111.3)$ & 19405.7 \\
4 & 13468.8 & 5002.4 & $(3664.2,23273.5)$ & 19609.3 \\
5 & 13529.4 & 5054.6 & $(3622.5,23436.3)$ & 19813.8 \\
6 & 13590.0 & 5107.0 & $(3580.4,23599.7)$ & 20019.4 \\
7 & 13650.9 & 5159.7 & $(3537.9,23763.8)$ & 20225.9 \\
8 & 13711.8 & 5212.6 & $(3495.0,23928.5)$ & 20433.5 \\
9 & 13772.9 & 5265.8 & $(3451.8,24093.9)$ & 20642.1 \\
10 & 13834.1 & 5319.3 & $(3408.3,24259.9)$ & 20851.6 \\
\hline
\end{tabular}




\section{Concluding remarks}

As pointed out by [8], discrete lifetimes have important applications. For example, actuaries and biostatisticians are interested in lifetimes of persons or organisms measured in months, weeks, or days. The reliability engineers use discrete lifetimes in modeling the number of defects in an equipment or the number of miles a plane has flown.

The log-convex property of the generalized Sibuya distribution studied in this paper, has various implications in reliability studies. In particular, it enables us to study the monotonic properties of various reliability functions. It also follows that generalized Sibuya distribution is infinitely divisible which has some important applications in probability theory.

\section{References}

[1] F. Alqallaf, M.E. Ghitany, M.F. Alfahad, A new two-parameter distribution with decreasing, increasing, bathtub hazard rate function and its applications, International Journal of Applied Mathematics, 33, No 3 (2020), 523548; DOI: 10.12732/ijam.v33i3.11.

[2] M. Bagnoli, T. Bergstrom, Log-concave probability and its applications, Economic Theory, 26 (2005), 445-469.

[3] J.A. Doornik, Object-Oriented Matrix Programming Using Ox, 3rd Ed., Timberlake Consultants Press and Oxford, London (2007).

[4] M.E. Ghitany, D.K. Al-Mutairi, F.A. Al-Awadhi, M.M. Al-Burais, Marshall-Olkin extended Lindley distribution and its applications, International Journal of Applied Mathematics, 25, No 5 (2012), 709-721.

[5] P.L. Gupta, Properties of reliability functions of discrete distributions, Communications in Statistics - Theory and Methods, 44 (2015), 4114-4131.

[6] P.L. Gupta, R.C. Gupta, R.C. Tripathi, On the monotonic properties of discrete failure rates, Journal of Statistical Planning and Inference, 65 (1997), 255-268.

[7] B.G. Hansen, On log-concave and log-convex infinitely divisible sequences and densities, The Annals of Probability, 16 (1988), 1832-1839.

[8] A.W. Kemp, Classes of discrete lifetime distributions, Communication in Statistics-Theory and Methods, 33 (2004), 3069-3093. 
[9] T.J. Kozubowski, K. Podgorski, A generalized Sibuya distribution, Annals of the Institute of Statistical Mathematics, 70 (2018), 855-887.

[10] C.D. Lai, M. Xie, Stochastic Ageing and Dependence for Reliability, 1st Ed., Springer Science + Business Media (2006).

[11] E.L. Lehmann, G. Casella, Theory of Point Estimation, Springer, New York (1998).

[12] F. Proschan, Theoretical explanation of observed decreasing failure rate, Technometrics, 5 (1963), 375-383.

[13] M. Sibuya, Generalized hypergeometric, digamma and trigamma distributions, Annals of the Institute of Statistical Mathematics, 31 (1979), 373390. 